\title{
ALEXANDRIA
}

Revista de Educação em Ciência e Tecnologia

ALEXANDRIA

\section{Aplicações para o Ensino de Equações Diferenciais}

\section{Applications for Teaching Differential}

\section{Sonia Barbosa Camargo Igliori ${ }^{\mathrm{a}}$; Marcio Vieira de Almeida ${ }^{\mathrm{a}}$}

a Departamento de Matemática, Pontifícia Universidade Católica de São Paulo, São Paulo, Brasil - sigliori@pucsp.br, marcioalmeidasp@gmail.com

\section{Palavras-chave:}

Ensino e aprendizagem do cálculo.

Diferenciabilidade. Equação diferencial. Educação matemática no ensino superior. GeoGebra.

\section{Keywords:}

Teaching and learning of calculus.

Differentiability.

Differential equation. Mathematics education at higher education. GeoGebra.
Resumo: Este artigo se insere no âmbito das pesquisas relacionadas ao ensino e à aprendizagem da Matemática no Ensino Superior, em especial o ensino e a aprendizagem do Cálculo Diferencial e Integral nos cursos de Exatas. Nele são apresentadas duas aplicações para a noção de solução de uma equação diferencial, referenciadas teoricamente pela noção de retidão local, formulada pelo pesquisador inglês David Tall. Na primeira é explorada a noção de campo de direções da equação. $\mathrm{Na}$ segunda é adaptada uma situação desenvolvida pelo pesquisador inglês para o esboço de uma curva solução de uma equação diferencial. A elaboração dessas aplicações toma por base a necessidade de integrar teoria e prática no campo da Educação Matemática e de produzir materiais para o ensino, baseados em resultados de pesquisas. Espera-se que tais aplicações favoreçam a formação, no aprendiz, de conceitos imagens ricos relativamente ao conceito matemático tratado.

Abstract: This paper fits the area of Mathematics Education at Higher Education, especially the area of studies concerning the teaching and learning of Differential and Integral Calculus. It is presented two applications for the differential equation solutions, theoretically referenced by the notion of local straightness, formulated by David Tall. The first application is explored the notion of slope field. The second one is adapted a situation developed by English researcher for a sketch of a solution curve of a differential equation. The development of these applications is based on the need to integrate theory and practice in the field of mathematics education and to produce materials for teaching, based on research results. It is expected that such applications to promote development of rich concepts images in the student, in the relation to the treaty mathematical concept. 


\title{
Introdução
}

Este artigo tem por alvo a investigação sobre o ensino e a aprendizagem do conceito de solução de uma equação diferencial por meio do desenvolvimento de aplicações produzidas no GeoGebra e referenciadas nos trabalhos de David Tall e seus colaboradores. E está fundamentado na seguinte questão: Como explorar diferentes representações do conceito de solução de equações diferenciais durante o processo de ensino?

É considerado o conceito de equação diferencial em virtude da importância dele para outras áreas do conhecimento, conforme pontuam Boyce e DiPrima:

\begin{abstract}
A importância das equações diferenciais está no fato de que mesmo as equações mais simples correspondem a modelos físicos úteis, como por exemplo, o decaimento de substâncias radioativas, o comportamento de sistemas de massas e molas e o comportamento de circuitos elétricos (BOYCE; DIPRIMA, 1999, prefácio).
\end{abstract}

E, Bassanezi (2013) destaca a importância das equações diferenciais como um tópico que pode ser abordado de maneiras diversas, dependendo do objetivo proposto. Esse pesquisador destaca o papel dessas equações para modelar um problema, quando diz que:

\footnotetext{
Um problema real não pode ser representado de maneira exata em toda sua complexidade por uma equação matemática ou um sistema de equações. Um modelo deve ser considerado apenas como um retrato ou uma simulação de um fenômeno e sua validação depende muito da escolha das variáveis e das hipóteses formuladas. É muito frequente em se tratando de modelar um fenômeno ou um experimento, obtermos equações para descrever as "variações" das quantidades (variáveis de estado) presentes e consideradas essenciais. Desta forma, as leis que regem tal fenômeno são traduzidas por equações de variações. Quando estas variações são instantâneas, a dinâmica do fenômeno se desenvolve continuamente e as equações matemáticas são denominadas equações diferenciais (BASSANEZI, 2013, p. 61 62).
}

Com relação ao ensino e aprendizagem das equações diferenciais, em Igliori e Oliveira (2013) foram apresentados resultados de um levantamento da produção acadêmica relacionada ao conceito, no período de 2000 a 2011. Nesse levantamento foram destacadas as seguintes dificuldades de ensino: esse vem acontecendo de modo que o foco é do desenvolvimento de soluções analíticas, obtidas a partir de manipulações algébricas sendo que emergem dificuldades de aprendizagem referentes à matemática básica, aos conceitos de derivada e integral e à intepretação de taxas de variação instantânea; em virtude de um modo em que é privilegiada a solução analítica, sem favorecer dessa forma a compressão do conceito de equação diferencial, dificultando a aplicação das equações em problemas contextualizados que exigem intepretação; dificuldades que os estudantes têm para pensar simultaneamente de modo algébrico e gráfico, apontadas em pesquisas (por exemplo, Artigue (1994)).

Como resultados, que apontam em direção favorecer a aprendizagem, Igliori e Oliveira (2013) destacam que: 
Para amenizar essas dificuldades, a maioria dos autores consultados apontou como possibilidade para o ensino de Equações Diferenciais o enfoque qualitativo do assunto, de forma contextualizada, a partir de situações-problema e favorecendo o equilíbrio entre a abordagem analítica, numérica e gráfica, por meio da utilização de recursos computacionais que auxiliem e agilizem o processo. Mas reforçam o cuidado com a escolha do programa computacional para o ensino do conteúdo, de modo a evitar que a atenção dos alunos se concentre em aprender a sintaxe do software, em vez de aprender o conteúdo matemático (IGLIORI; OLIVEIRA, 2013, p. 21).

As aplicações ${ }^{1}$ propostas foram produzidas de acordo com as direções apontadas por Igliori e Oliveira, que sugerem um enfoque qualitativo ao estudo das Equações Diferenciais procurando favorecer o equilíbrio entre as abordagens analítica, numérica e gráfica, corroborando as indicações apresentadas em Artigue (1994).

Esse equilíbrio é favorecido com a utilização do software, de Geometria Dinâmica, GeoGebra, pois ele possui interface simples e intuitiva e possibilita trabalhar conjuntamente a Geometria, a Álgebra e o Cálculo. Tal software é munido das ferramentas necessárias para o desenvolvimento das aplicações, não necessita de computadores "poderosos" e possui uma versão para dispositivos móveis (como tablets com sistemas Android, IOS e Windows) e, para smartphones (com sistema Android ${ }^{2}$ ) e possibilita a elaboração e modificação de applets, tanto para uso em sala de aula quanto para disponibilizar em sitios da internet e na plataforma GeoGebra Tube ${ }^{3}$.

No GeoGebra estão reunidos elementos que pesquisadores, do campo da Educação Matemática, apontam como salutares quando computadores são utilizados no ensino, como, por exemplo, garantir a visualização de duas (ou mais) representações de conceitos matemáticos. Segundo Tall (1991), desconsiderar o papel da visualização na aprendizagem da Matemática "é negar as raízes de muitas de nossas ideias matemáticas mais profundas" (TALL, 1991, p. 1, tradução nossa).

Para Guzmán, a visualização não auxilia

[...] apenas no nascimento do pensamento matemático, mas também na descoberta de novas relações entre objetos matemáticos e, certamente, em processos de transmissão e comunicação que são a própria atividade matemática (GUZMÁN, 2002, p. 2 - 3, tradução nossa).

Outro ponto que merece ser ressaltado é a possibilidade de apresentar múltiplas representações de um objeto matemático. Para Gravina e Santarosa (1998), como relação

[...] ao potencial das múltiplas representações, considerando que um mesmo objeto matemático pode receber diferentes representações e que estas registram diferentes facetas do mesmo, uma exploração que transita em diferentes sistemas torna-se significativa no processo de construção do conceito. Por exemplo, a uma função pode-se associar uma representação gráfica que evidencia variações qualitativas, ou

\footnotetext{
${ }^{1}$ Neste artigo chamamos aplicação, o tratamento informático de uma atividade para o ensino.

2 Até o momento da escrita do artigo, março de 2016. Contudo, é de conhecimento dos autores que a versão para smartphones com sistema IOS está em desenvolvimento.

${ }^{3}$ Endereço eletrônico: https://tube.geogebra.org/?lang=pt_BR.
} 
uma representação matricial numérica que evidencia variações quantitativas, ou ainda um fenômeno cujo comportamento é dado pela função. Ou ainda, pode-se estudar família de funções sob o ponto de vista de operações algébricas e correspondentes movimentos geométricos nos gráficos associados (GRAVINA, SANTAROSA, 1998, p.11).

As aplicações desenvolvidas compõem o resultado parcial de uma pesquisa mais ampla que visa o desenvolvimento de recursos ${ }^{4}$ para o ensino do Cálculo Diferencial e Integral. A construção desse exemplo se insere na seguinte problemática: a necessidade de integrar teoria e prática no campo da Educação Matemática e de produzir materiais para o ensino, em especial do Cálculo, baseados em resultados de pesquisas.

Essa problemática tem merecido a atenção de pesquisadores, do campo da Educação Matemática. Rasmussen et al. ressaltam que "é fundamentalmente importante que o corpo de pesquisa em ensino, aprendizagem e entendimento do Cálculo contribua com a prática educacional de estudantes que estão matriculados em cursos de Cálculo a cada ano" (RASMUSSEN et al., 2014, p. 507, tradução nossa).

Jaworski, por exemplo, diz que esse campo,

[...] tem se tornado maduro em suas considerações teóricas. Entretanto, a posição do ensino da Matemática permanece teoricamente anômala e não desenvolvida. Enquanto teorias proveem-nos lentes para analisar o ensino (LERMAN, 2001), as "grandes teorias" não parecem oferecer percepções claras para o ensino e maneiras nas quais o ensino vise à promoção da aprendizagem na Matemática (JAWORSKI, 2006, p. 188, tradução nossa).

Essas reflexões reforçam a importância de criar atividades para ensino, fundamentadas teoricamente, foco deste trabalho.

\section{Referencial teórico}

As aplicações apresentadas neste artigo são referenciadas em elementos teóricos de Tall e seus associados. As aplicações desenvolvidas estão no âmbito de uma abordagem sensível do Cálculo que, segundo Tall

[...] é construída na evidência de nossos sentidos humanos e utiliza esses insights como uma base significativa para vários desenvolvimentos posteriores, do cálculo prático para aplicações para o desenvolvimento teórico na analise matemática e até a abordagem lógica na utilização dos infinitesimais. (TALL, 2010, p. 1, tradução nossa).

O computador pode contribuir para o desenvolvimento de atividades com as características descritas por Tall, visto que por meio de um software adequado é possível desenvolver atividades para um dado domínio de conhecimento levando em consideração os obstáculos conhecidos e procurando resolver eventuais conflitos cognitivos de forma adequada (TALL, 1986, p. 71). A potencialidade da utilização dos computadores no ensino

\footnotetext{
${ }^{4} \mathrm{O}$ termo recurso é utilizado no sentido utilizado por Trouche e Gueudet (2009).
} 
dos tópicos avançados da Matemática para a aprendizagem é reforçada pelo pesquisador inglês, quando diz que é possível

[...] utilizar os computadores para visualizar conceitos matemáticos de maneira útil seja no Cálculo e na Análise. A utilização criativa de um software, que plota gráficos, e de calculadoras gráficas tem permitido aos estudantes lidar de maneira significativa com conceitos como a diferenciação por meio da noção de retidão local, integração por meio da soma de áreas, e resolver equações diferenciais (de $1 .^{a}$ ordem) por meio da visualização da construção das curvas solução com um gradiente dado. Durante esse tempo, me tornei cada vez mais consciente do conceito imagem limitado oferecido por gráficos plotadores de gráficos que só desenham gráficos razoavelmente suaves dados por fórmulas (TALL, 1993, p. 2, tradução nossa).

Nessa perspectiva, um computador, munido de um software adequado, pode ser utilizado "para propiciar imagens que auxiliarão no desenvolvimento de tópicos do Cálculo e da Análise" (ALMEIDA, 2013, p. 114).

Em Tall (2000) foi apresentada outra característica que determinados ambientes computacionais possuem e pode ser utilizada para o desenvolvimento cognitivo dos aprendizes. Tall diz que os computadores:

[...] podem executar quaisquer algoritmos de forma rápida e eficiente, além de exibir o resultado final com uma gama de diferentes representações. Por exemplo, os resultados podem ser representados visualmente e manipulados fisicamente. Utilizando um mouse é possível ao estudante construir relações corporificadas que fazem parte de uma estrutura conceitual mais rica e ampla (TALL, 2000, p. 10, tradução nossa).

As aplicações, como as apresentadas neste artigo, são denominadas por esse pesquisador como um organizador genérico. Um organizador genérico é definido como "um ambiente (ou micromundo) que permite ao aprendiz manipular exemplos e (se possível) contraexemplos de um conceito matemático específico ou de um sistema de conceitos relacionados" (TALL, 2000, p. 10, tradução nossa, grifo do autor). O pesquisador considera parte de desenvolvimento de abordagens devem ser utilizados organizadores genéricos, pois elas "dão ao aprendiz experiências apropriadas de modo que ele está cognitivamente pronto para novos conceitos matemáticos quando eles são introduzidos” (TALL, 1986, p. 5, tradução nossa).

Para o desenvolvimento de um organizador genérico é necessário selecionar uma ideia importante e essencial, que será o foco da atenção do estudante. Essa não é, necessariamente, fundamental para a teoria matemática, porém, ela auxilia o sujeito a desenvolver intuições apropriadas ao desenvolvimento teórico. Segundo essas características, Tall formulou a noção de raízes cognitivas como:

[...] uma unidade cognitiva que é (potencialmente) significativa ao estudante naquele momento, no entanto deve conter sementes de uma expansão cognitiva para definições formais e desenvolvimento teórico futuro (TALL, 2000, p. 11, tradução nossa). 
Uma raiz cognitiva é a noção de retidão local. Essa é definida por Tall como uma raiz cognitiva apropriada para o conceito de derivada e que será utilizada para o conceito de equação diferencial nas aplicações propostas. Essa está baseada na percepção de quanto maior a ampliação menor será a curvatura percebida, por exemplo, num objeto (TALL, 1989). Tal noção seria apropriada ao conceito de derivada, pois ela "permite que a inclinação da função seja vista como a mudança de inclinação do próprio gráfico" (TALL, 2000, p. 11, tradução nossa, grifo do autor) e para o conceito de equação diferencial é que nesta ideia podemos conjecturar qual seria o esboço de uma curva solução de uma dada equação. Nesse sentido, a representação gráfica de função diferenciável, quando ampliada em determinada porção, assemelha-se localmente a um segmento de reta. Observe a Figura 1, nela é possível perceber que uma curva diferenciável, localmente, assemelha-se a um segmento de reta:

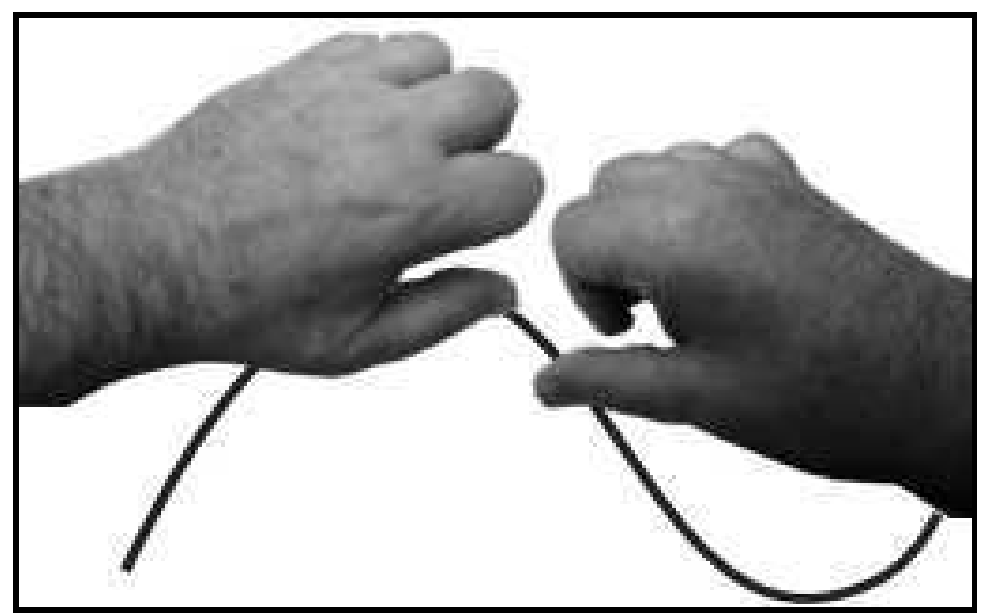

Figura 1 - Uma pequena parte da curva assemelhasse a um segmento de reta Fonte: TALL, 2010, p. 11

Tall diferencia a retidão local da noção de linearidade local, essa última tem sido utilizada em cursos modernos de Cálculo, para a introdução do conceito de derivada, do seguinte modo:

\begin{abstract}
A linearidade local é uma concepção simbólica, procura a fórmula para descrever a função inclinação com um grau de precisão apropriado. Retidão local é uma corporificação primitiva que pode prover uma corporificação humana subjacente à função inclinação. No meu Graphic Calculus original, utilizei a noção de retidão local para dar uma imagem mental sobre se uma função é diferenciável ou não, dependendo do que aconteceu sob uma grande ampliação (é que se torna eventualmente localmente reta ou não). Isso deu uma primeira indicação da noção de diferenciabilidade e não-diferenciabilidade que não está contemplado nos cursos tradicionais. Além disso, a abordagem da retidão local prove uma nova habilidade a capacidade de olhar para um gráfico que é imaginado para ser localmente reto e visualizar suas derivadas (TALL, 2010, p. 487, tradução nossa).
\end{abstract}

Em outro artigo (TALL, 2001) é destacada a importância dos aspectos sensóriomotores e visuais, na composição do pensamento matemático e que esses aspectos atuam, de maneira importante, numa interface na qual o computador é utilizado. Por meio de ações simples, como, por exemplo, clicar em determinado local ou utilizar o teclado para atribuir 
um valor a uma variável, se pode fornecer suporte para o desenvolvimento de conceitos teóricos de alto nível (TALL, 2001).

\section{Aplicações para o ensino de equação diferencial}

Nesta seção passamos a apresentar as aplicações elaboradas pelos autores deste artigo.

A primeira refere-se à representação do campo de direções associado a uma equação diferencial. Destaca-se que a utilização do campo de direção para o estudo das soluções das equações diferenciais já é abordada em livros de Cálculo, editados atualmente, como Stewart (2005) e Anton, Bivens e Davis (2007). Para exemplificar, no capítulo 9 de Stewart (2005), após apresentar a definição de uma equação diferencial, a ordem de uma equação diferencial e dois exemplos de modelagem, cujo modelo é uma equação diferencial, o autor apresenta os campos de direção como uma maneira pela qual é possível "aprender muito sobre a solução [de uma equação diferencial] através de uma abordagem gráfica (campos de direção)" (STEWART, 2005, p. 586, adaptado), mesmo quando não é possível obter uma solução analítica da equação diferencial.

Considerando a seguinte variação do comando CampoDeDireções:

CampoDeDireções $[<\mathrm{f}(\mathrm{x}, \mathrm{y})>$, $<$ Número $\mathrm{n}>$, $<$ Fator de Escala a $>,<$ Min $\mathrm{x}>,<$ Min $\mathrm{y}>,<$ Max $\mathrm{x}>,<\operatorname{Max} \mathrm{y}>$ ]

Em que cada um dos parâmetros utilizados são: $\langle\mathrm{f}(\mathrm{x}, \mathrm{y})>$ é a sentença da equação diferencial dada na forma normal, ou seja, $\frac{d y}{d x}=f(x, y)$; $<$ Número n> é a quantidade de segmentos que aparecerão na Janela de Visualização e no subconjunto de $\mathbb{R}^{2}$ definido, o padrão desse comando é 40 segmentos; <Fator de Escala a $>$ é um número entre 0 e 1 que determina o tamanho que cada segmento do campo terá; $<$ Min $\mathrm{x}>$, $<$ Min $\mathrm{y}>$, $<\operatorname{Max} \mathrm{x}>$, $<\operatorname{Max} \mathrm{y}>$ são pontos da fronteira de um subconjunto do plano cartesiano, no qual o campo de direções será esboçado na Janela de Visualização.

Para exemplificar a utilização desse comando, considere a equação diferencial $y^{\prime}=2 x$, esboçando o campo de direções associado a ela, no conjunto $\left\{(x, y) \in \mathbb{R}^{2} \mid-2 \leq x \leq 2\right.$ e $0 \leq y \leq 4\}$. Abaixo segue o comando que deve ser digitado no campo Entrada, do GeoGebra: CampoDeDireções[2x, 10, 0.5, -2, 0, 2, 4]. O campo de direções é apresentado na Figura 2: 


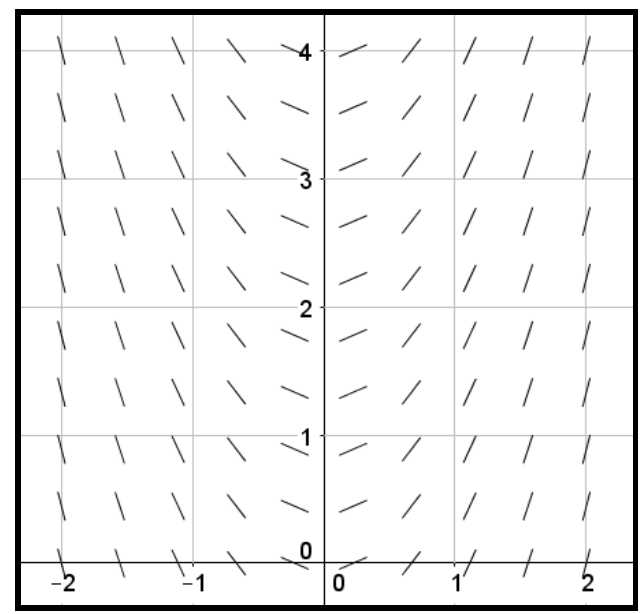

Figura 2 - Campo de direções da equação diferencial

Fonte: Os autores

Na aplicação apresentada é utilizado esse comando e são definidos controles deslizantes para os parâmetros: $<$ Número $\mathrm{n}>$, $<$ Fator de Escala $\mathrm{a}>$, $<$ Min $\mathrm{x}>$, $<$ Min $\mathrm{y}\rangle$, $<$ Max $\mathrm{x}>$, <Max y>. Na Figura 3 está apresentada a aplicação em que foi esboçado o campo de direções associado a equação diferencial $\frac{d y}{d x}=9,8-\frac{y}{5}$ no seguinte conjunto do plano $\{(x, y)$ $\in \mathbb{R}^{2} \mid-1 \leq x \leq 9$ e $\left.45 \leq y \leq 55\right\}$

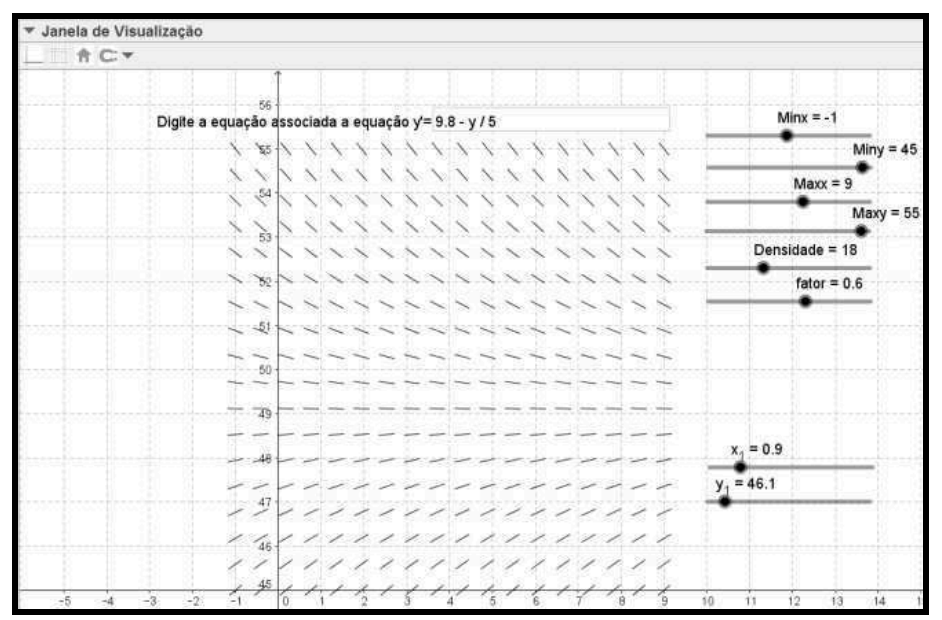

Figura 3 - Aplicação que representa o campo de direções de uma equação diferencial em um subconjunto de $\mathbb{R}^{2} 1$

Fonte: Os autores

A segunda aplicação proposta é inspirada na seguinte situação problema proposta por Tall:

Considere o problema inverso da diferenciação (Não, esse não é a integração!). $O$ problema é o seguinte - se você conhece a inclinação de uma função em qualquer ponto, como poderíamos construir o gráfico que tem essa inclinação? (TALL, 2000, p. 14, tradução nossa).

E, ele atribui o significado corpóreo para o conceito das equações:

Se eu apontar meu dedo em um ponto $(x, y)$ qualquer do plano, então eu posso calcular a inclinação da curva solução naquele ponto como $m=F(x, y)$ e traçar um segmento de reta pequeno com inclinação igual a $m$ através do ponto $(x, y)$ (TALL, 2000, p. 14, tradução nossa). 
Para a utilização do significado corporificado proposto, o pesquisador desenvolveu uma aplicação que constrói a solução gráfica de uma equação diferencial de $1^{\mathrm{a}}$ ordem do seguinte modo: o mouse é utilizado para mover um segmento, cuja inclinação é definida pela equação diferencial, dada pelo usuário, e com um clique sobre o plano cartesiano, esse segmento é fixado (BLOKLAND et al., 2000 apud TALL, 2001, p. 211). Na Figura 4 está representada a tela desse software:

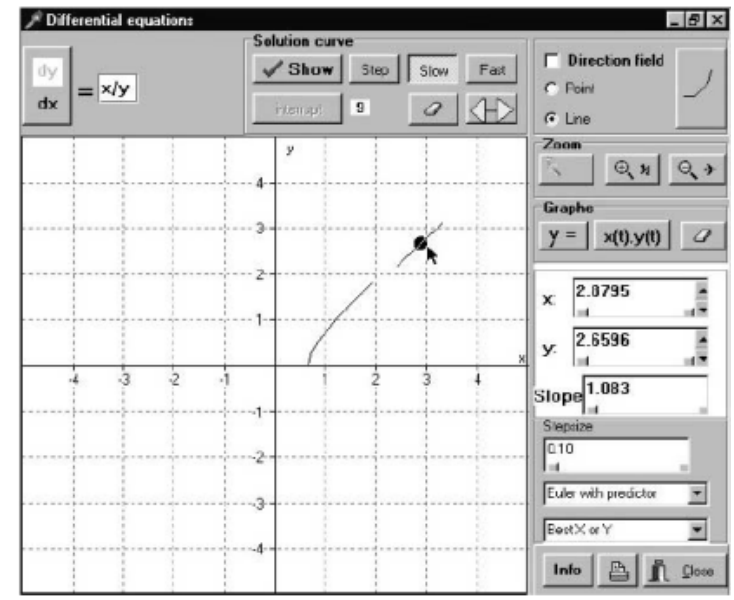

Figura 4 - Exemplo de software que explora a solução de uma equação diferencial

Fonte: BLOKLAND et al. (2000 apud TALL, 2001, p. 211).

Para construir essa função no GeoGebra foi necessário construir uma ferramenta chamada Equação Diferencial, que tem como parâmetros uma função $f$ de várias variáveis (que será a equação diferencial considerada) e um ponto P. Ela constrói um segmento que tem como ponto médio o ponto $P$, e como inclinação o valor numérico de $f$ em $P$. A Figura 5 representa essa aplicação para o caso da equação diferencial $\frac{d y}{d x}=x^{2}+x$ :

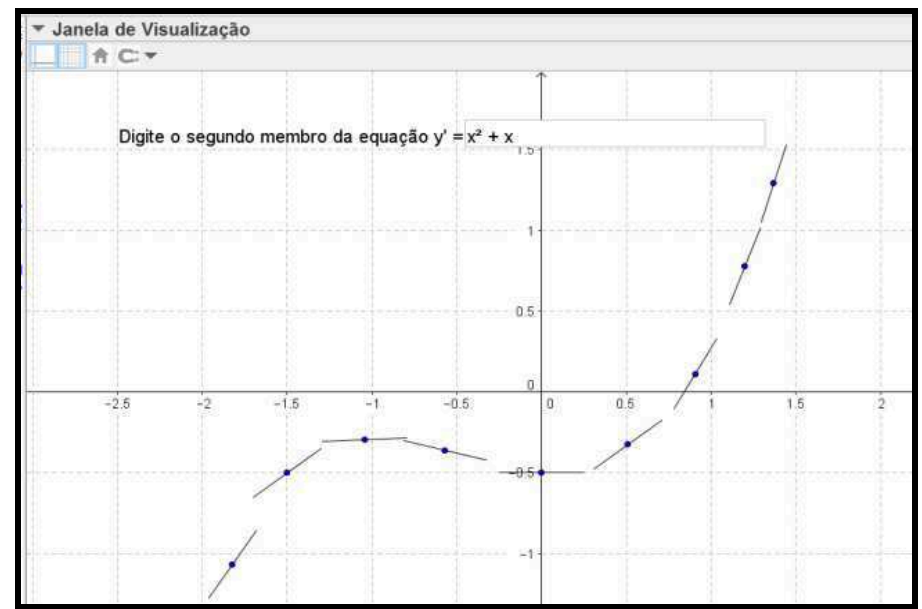

Figura 5 - Aplicação que esboça segmentos para explorar a solução de uma equação diferencial Fonte: Os autores.

O GeoGebra permite a construção de ferramentas personalizadas, como aquela que construiu os segmentos apresentados na Figura 5. Para a construção desse tipo de ferramenta precisamos fazer uma construção inicial que será a rotina executada pela ferramenta. Para 
isso, precisamos fazer a construção um segmento que tem como ponto médio o ponto $P$, e como inclinação o valor numérico de $f$ em $P$. Com os seguintes passos desenvolvemos a referida construção:

1) Digite no campo de Entrada uma função de duas variáveis, que denominaremos por $f(x, y)$;

2) Digite no campo de Entrada: $P=(1,1)$ para construir um ponto no plano;

3) Nesse passo iniciaremos a construção do segmento. Esse segmento possuirá medida fixa, igual a 0,25 unidades de medida de comprimento padrão do GeoGebra. Para a ferramenta é necessário construir o segmento, por meio dos seguintes passos: construímos uma reta que passa pelo ponto $P$ e tem inclinação igual a $f(P)^{5}$; em seguida, uma circunferências com centro em $P$ e raio igual a 0,25 , com a ferramenta "Círculo dados centro e raio"6 é construída; as interseções da circunferência com a reta são os pontos procurados; por fim, utilizamos a ferramenta "Segmento" e obtemos o segmento procurado.

Com a construção feita, menu principal, clique em "Ferramenta" e selecione a opção "Criar nova ferramenta". Com essa opção selecionada, aparecerá uma caixa de diálogo (Figura 6), com a qual definiremos a nova ferramenta.

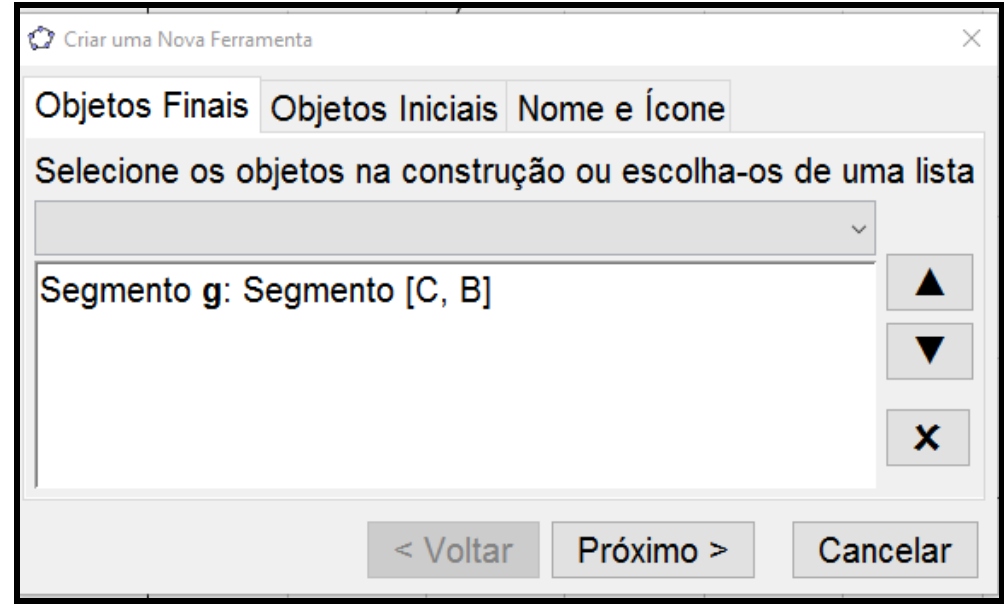

Figura 6 - Caixa de diálogo para a criação de uma ferramenta Fonte: Os autores.

Como "Objetos Finais" deve ser selecionado o "Segmento g" construído anteriormente; e como "Objetos Iniciais" devem ser selecionados o "Ponto P" e a "Função de várias variáveis f". Um último passo é a determinação do nome e do ícone. Com isso, basta selecionar um ponto e uma função de duas variáveis para construir o segmento que tem como

\footnotetext{
${ }^{5}$ No GeoGebra para fazer isso é necessário digitar o seguinte no campo Entrada: $\mathrm{y}-$ $\mathrm{y}(\mathrm{P})=\mathrm{f}(\mathrm{x}(\mathrm{P}), \mathrm{y}(\mathrm{P}))^{*}(\mathrm{x}-\mathrm{x}(\mathrm{P}))$. Sendo que o comando $\mathrm{x}(\mathrm{P})$ é o valor da abcissa do ponto $\mathrm{P} ; \mathrm{y}(\mathrm{P})$ é a ordenada do ponto $\mathrm{P}$ e $\mathrm{f}(\mathrm{x}(\mathrm{P}), \mathrm{y}(\mathrm{P}))$ é o valor de $f(P)$.

${ }^{6} \mathrm{O}$ ícone da ferramenta é o seguinte:

${ }^{7} \mathrm{O}$ ícone da ferramenta é o seguinte:
} 
ponto médio o ponto $P$, e como inclinação o valor numérico de $f$ em $P$. Na Figura 5 foi utilizada essa ferramenta para a equação diferencial $\frac{d y}{d x}=x^{2}+x$.

\section{Considerações finais}

Neste trabalho foram apresentadas duas aplicações desenvolvidas no GeoGebra para o ensino de equações diferenciais. A primeira utiliza campos de direção associado a uma equação diferencial e a outra está inserida nos estudos que têm por base o significado corpóreo de um conceito matemático desenvolvido por Tall (2000).

Essas duas aplicações estão em consonância com os resultados apresentados por Igliori e Oliveira (2013), pois nelas é possibilitado um equilíbrio entre as abordagens analíticas, numéricas e gráficas do conceito de equação diferencial e utiliza-se de um recurso computacional que objetiva focar no desenvolvimento conceitual das equações diferenciais. Também com as indicações de Anton e outros (2007) quando sugerem ao professor, que ministra uma disciplina de Cálculo, que faça uso de livros on-line, apresentações em PowerPoint e simulações interativas para exemplificar a matéria, estimular o aprendizado e o interesse dos alunos,

As aplicações desenvolvidas neste artigo podem auxiliar o professor a desenvolver, no aprendiz, conceitos imagens ${ }^{8}$ ricos relativamente ao conceito matemático em questão, na medida em que favorece a exploração de outros tipos de representação para as equações diferenciais e não apenas o algébrico. Com vistas a promover, o desenvolvimento do conceito de equação diferencial, de acordo com os níveis de realidade, proposto por Pires (2002), pois

[...] ninguém promove a aprendizagem de conteúdos que não domina nem a constituição de significados que não possui ou a autonomia que não teve oportunidade de construir (PIRES, 2002, p. 48).

Dessa forma, as aplicações propostas contemplam a necessidade, proposta por Pires, de compreender o conceito de equação diferencial, de forma que possibilite ao aluno a oportunidade de refletir o conceito de equações diferenciais, a parir de outras representações e de problemas relacionados ao cálculo, em situações de aplicações do conceito, e em outros espaços educativos.

Com relação à utilização desses materiais de ensino, ressaltamos que até o presente momento, não foi feito nenhuma exploração e aplicação desses com estudantes, para testar a efetividade, ou não desse material. Contudo, é entendido que a discussão teórica sobre a produção de materiais de ensino, produzidos com bases em resultados do campo de pesquisa

\footnotetext{
${ }^{8}$ O termo conceito imagem é utilizado no sentido de Vinner e Tall (1981).
} 
da Educação Matemática, se faz necessária com vistas a promover uma integração entre resultados teóricos e as práticas de ensino.

Este trabalho insere-se em uma pesquisa mais ampla, que tem por objetivos colocar à disposição dos professores materiais para o ensino de Cálculo, cuja construção é embasada em resultados de pesquisa, e contribuir com o estreitamento da relação teoria e prática.

\section{Referências}

ALMEIDA, M. V. Um Panorama de Artigos sobre a Aprendizagem do Cálculo Diferencial e Integral na Perspectiva de David Tall. Dissertação de Mestrado em Educação Matemática Pontifícia Universidade de São Paulo, São Paulo, 2013.

ANTON, H.; BIVENS, I.; DAVIS, S. Cálculo. Volume I. Tradução Claus Ivo Doering. Bookman, 8a ed. Porto Alegre: Bookman, 2007.

ARTIGUE, M. Didactical engineering as a framework for the conception of teaching products. In: BIEHLER, R.; SCHOLZ, R. W.; WINKLEMANN, B. Didactics of mathematics as a scientific discipline. Dordrecht: Kluwer Academic Publishers, 1994. p. 27-39.

BASSANEZI, R. C. Temas \& Modelos. Santo André: UFABC, 2013.

BOYCE, W. E.; DIPRIMA, R. C. Equações Diferenciais Elementares e Problemas de Valores de Contorno. Tradução de Horácio Macedo. 6. ed. rev. Rio de Janeiro: LTC, 1999.

GUEUDET, G.; TROUCHE, L. Towards new documentation systems for mathematics teachers?. Educational Studies in Mathematics, v. 71, n. 3, p. 199-218, 2009.

GUZMÁN, M. The role of visualization in the teaching and learning of mathematical analysis. In: INTERNATIONAL CONFERENCE ON THE TEACHING OF MATHEMATICS AT THE UNDERGRADUATE LEVEL, 2, 2002, Hersonissos. Proceedings of $2^{\text {nd }}$ International Conference on the Teaching of Mathematics at the Undergraduate Level. Hersonissos: University of Crete, 2002, p. 1-24.

JAWORSKI, B. Theory and practice in mathematics teaching development: Critical inquiry as a mode of learning in teaching. Journal of Mathematics Teacher Education, 9, p. 187-211, 2006.

IGLIORI, S. B. C.; OLIVEIRA, E. A.. Ensino e aprendizagem de equações diferenciais: um levantamento preliminar da produção científica. EM TEIAI Revista de Educação Matemática e Tecnológica Iberoamericana, v. 4, n. 2, 2013.

RASMUSSEN, C.; MARRONGELLE, K.; BORBA, M. C. Research on calculus: what do we know and where do we need to go?. ZDM, v. 46, n. 4, p. 507-515, 2014.

SANTAROSA, L. M. A.; GRAVINA, M. aprendizagem da matemática em ambientes informatizados. In: IV CONGRESSO RIBIE. Brasília. 1998.

STEWART, J. Cálculo. Vol. II. 4 ed. São Paulo: Pioneira Thomson Learning, 2005. 
TALL, D. Building and Testing a Cognitive Approach to the Calculus Using Interactive Computer Graphics. 1986. Tese de Doutorado em Educação Matemática - University of Warwick, Inglaterra, 1986.

TALL, D. Concept images, computers, and curriculum change. For the Learning of Mathematics, v 9, n 3, p. 37-42, 1989.

TALL, D. Intuition and rigour: the role of visualization in the calculus. In. ZIMMERMAN, W; CUNNINGHAN, S. (Eds) Visualization in teaching and learning mathematics, v. 19, p. 105-119, 1991.

TALL, D. Real Mathematics, Rational Computers and Complex People. In: ANNUAL INTERNATIONAL CONFERENCE ON TECHNOLOGY IN COLLEGE MATHEMATICS TEACHING, 5., 1993, Proceedings..., Addison-Wesley, p. 243 - 258, 1993.

TALL, D. Biological Brain, Mathematical Mind \& Computational Computers (how the computer can support mathematical thinking and learning). In: ASIAN TECHNOLOGY CONFERENCE IN MATHEMATICS, 5, 2000, Chiang Mai. Proceedings... Blackwood: ATCM Inc, 2000.

TALL, D. Cognitive development in advanced mathematics using technology. Mathematics Education Research Journal, v.12, n.3, p. 210-230, 2001. Disponível em $<$ http://homepages.warwick .ac.uk/staff/David.Tall/pdfs/dot2001b-merj-amt.pdf>. Acesso em: 18 fev. 2015.

TALL, D. A sensible approach to the calculus. In: PLUVINAGE, F.; CUEVAS, A. (Eds.), Handbook on calculus and its teaching. México: Pearson. 2010. Disponível em: <http://homepages.warwick.ac.uk/staff/David.Tall/pdfs/dot2012z-sensible-calculus.pdf>. Acesso em: 14 mar. 2015.

VINNER, S.; TALL, D. Concept image and concept definition in mathematics with particular reference to limits and continuity. Educational studies in mathematics, v. 12, n. 2, p. 151-169, 1981.

\section{SOBRE OS AUTORES}

SONIA BARBOSA CAMARGO IGLIORI. Realizou estágio de pós doutorado na Université Paris VII, França, de 1995 a 1996, com Michèle Artigue. Doutorou-se em Matemática pela PUC-SP em 1986, na área de Análise Funcional e teve orientação de Domingos Pisaneli. É professora titular da PUC-SP. É professora permanente do Programa de Estudos Pós Graduados em Educação Matemática, tendo sido coordenadora desse Programa de 1995 a 2005, e no biênio 2011-2013. Foi editora da revista "Educação Matemática Pesquisa" e atualmente é editora da revista "Ensino de Matemática em Debate". É uma das criadoras do GT 19 de Educação Matemática da ANPEd, tendo sido a primeira coordenadora. É membro do GT 4 do "Ensino Superior" da Sociedade Brasileira de Educação Matemática. É líder do grupo de pesquisa do CNPq denominado: "O elementar e o superior em Matemática" e, até o momento, orientou 6 teses de doutorado e co-orientou 2; orientou 30 dissertações de mestrado sendo 27 em Educação Matemática e 3 em Matemática, e 11 projetos de Iniciação Científica. Publicou capítulos de livros e artigos em periódicos nacionais e internacional. 
Atualmente assume a função de Chefe do Departamento de Matemática da PUC/SP, gestão 2013-2015.

MARCIO VIEIRA DE ALMEIDA. Possui graduação em Licenciatura em Matemática pela Universidade de São Paulo (2009), mestre em Educação Matemática pela Pontifícia Universidade Católica de São Paulo (2013) e atualmente doutorando do Programa de Estudos Pós-graduados em Educação Matemática da PUC/SP. Tem experiência na área de Matemática, com ênfase em Matemática, atuando principalmente nos seguintes temas: ensino de cálculo, cálculo diferencial e integral, aprendizagem do Cálculo Diferencial e Integral.

Recebido: 02 de maio de 2016.

Revisado: 06 de setembro de 2016.

Aceito: 01 de novembro de 2016. 